\title{
Optical Techniques to Feed and Control GaAs MMIC Modules for Phased Array Antenna Applications
}

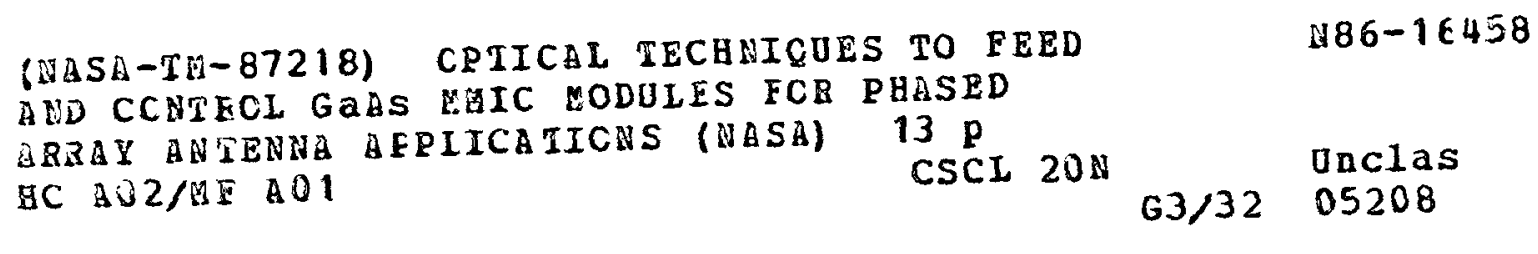

K.B. Bhasin, G. Anzic,

R.R. Kunath, and D.J. Connolly

Lewis Research Center

Cleveland, Ohio

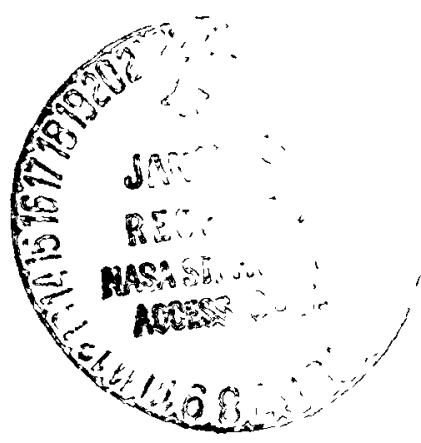

Prepared for the

11th Annual Communications Satellite Systems Conference

sponsored by the American Institute of Aeronautics and Astronautics

San Diego, California, March 16-20, 1986 
OPTICAL TECHNIQUES TO FEED AND CONTROL GaAS MMIC MODULES FOR PHASED ARRAY ANTENNA APPLICATIONS

\author{
K.B. Bhasin, G. Anzic, R.R. Kunath, and D.J. Connolly \\ National Aeronautics and Space Administration \\ Lewis Research Center \\ Cleveland, Ohio 44135
}

\section{Abstract}

A complex signal distribution system is required to feed and control GaAs monolithic microwave integrated circuits (MMICS) for phased array antenna applications above $20 \mathrm{GHz}$. Each MMIC module will require one or more RF lines, one or more bias voltage lines, and digital lines to provide a minimum of 10 bits of combined phase and gain control information. In a closely spaced array, the routing of these multiple lines presents difficult topology problems as well as a high probability of signal interference. To overcome GaAs MMIC phased array signal distribution problems, optical fibers interconnected to monolithically integrated optical components with GaAs MMIC array elements are proposed as a solution. System architecture considerations using optical fibers are described. The analog and digital optical links to respectively feed and control MMIC elements are analyzed. It is concluded that a fiber optic network will reduce weight and complexity, and increase reliability and performance, but higher power will be required.

\section{Introduction}

In an effort to achieve rapid beam reconfigurability and steering in future space communications systems, the use of phased array antennas, either directly radiating, or in feed systems, has been under investigation. 1 Prompting this move is the increased ability of GaAs monolithic microwave integrated circuits (MMICs) to provide lightweight, low loss beam forming networks with the necessary varjable amplitude and phase shifting capabilities.? Unfortunately the conventional cabling used to furnish bias and control signals to MMICs results in a complex signal distribution network. Fiber optic technology offers promise to improve signal distribution for GaAs MMIC phased arrays.

While other optical techniques for providing phase and amplitude excitation of phased arrays exist, 3-5 a natural extension is to retrofit the MMICs with optical integrated circuits (0ICs) to exploit the advantages of optical interconnection for a signal distribution network. This is feasible since MMICs and OICs can be monolithically integrated on a single GaAs chip.

On a systems level, optical control of MMICs offers advantages in the following areas. Current MMIC arrays use complex and expensive mounting fixtures. These fixtures adapt the MMIC to a waveguide environment by providing an interface between the MMIC and the external RF and control signal sources. Even though small (approximately $3 \times 1 \times 1 \mathrm{in}$. at $30 \mathrm{GHz}$ ), the fixtures are bulky and limit array spacing to a minimum of approximately two times the wavelength. An experimental MMIC phased array antenna, using conventional signal distribution techniques, is shown in Fig. 1 .
Control signal connection to the fixture is achieved with multiconductor cable or by specialized flexible printed circuit boards. Standard connectors normally used with this cable are 111suited because space is limited. Instead, special clamping connectors or custom printed circuit board adapters are used to access the fixtures; thrs limits the interelement clearance needed to the printed circuit board or ribbon cable thickness (approx. 1/16 in.).

These limitations can be overcome with the use of an optical fiber interconnection network. A single multiplexed optical fiber could be used to transmit $R F$ and control signals, eliminating the complex mounting fixtures and waveguide hardware. By interfacing to a microstrip environment rather than waveguide, array spacings of as little as $\lambda / 2$ could be achieved, where $\lambda$ is the operating wavelength. The additional benefits of low weight, minumum cross-talk, flexibility and immunity to electromagnetic interference are also offered by fiber optic signal distribution techniques. The disadvantages are the power required for electrical to optical conversion or vice-versa and the dynamic range limitations of optical components.

In this paper, we discuss the system design considerations for GaAs MMIC based phased array antennas and various optical techniques for its signal distribution. The RF optical link for analog signal transmission and digital optical link for phase and gain control of MMICs are also presented. Associated optical components required to achieve the signal distribution network are highlighted.

\section{System Design Considerations}

The system design presented is applicable to planar arrays used either as feeds to a reflector based antenna system or as a direct radiating array. Each array element needs optical connections for RF and control/data signals. It is desirable to have all these signals on a single multimode fiber, however, state-of-the-art RF modulation techniques require specialized optical components and therefore independent cabling. A conceptual diagram of the optical distribution network for an MMIC based phased array is shown in Fig. 2.

The heart of each array element is an MMIC transmit or receive module possessing a 5-bit variable phase shifter, a 4 or 5 bit variable power amplifier, and an integral D/A converter to provide the required analog bias voltages. Characteristics of MMICs being developed for 20 to 30 $\mathrm{GHz}$ comunications satellites are discussed below:

\section{GaAs MMIC for 20 to $30 \mathrm{GHz}$ System}

NASA Lewis Research Center has a substantial, on-going program to develop MMIC circuits in the 20 and $30 \mathrm{GHz}$ frequency bands primarlly for phased 
array antenna applications. The features of these MMICs which will play a key role in the optical signal'distribution network development are:

Variable Phase Shifter (VPS)

The VPS module functions include a phase shifter circuit with controls that provide a digitally selectable module phase shift capability of 0 to $360^{\circ}$ in increments of $11.25^{\circ}$. A two-stage buffer amplifier follows the phase shifter to compensate for the phase shifter losses and a threestage power amplifier completes the module. Each submodule requires a minimum of five connections for phase shifting and several bias connections. More detailed information on this VPS module is given elsewhere.6

\section{Variable Power Amplifier (VPA)}

These can be electronically switched to any one of five output power levels: $500,125,50$, 12.5 , and $0 \mathrm{~mW}$. The efficiency is to vary from 15 percent at the $500 \mathrm{~mW}$ level to 6 percent at the $12.5 \mathrm{~mW}$ leve1. The VPA consists of a four-stage dual gate FET amplifier and a D/A converter on a $3.05 \times 6.45 \mathrm{~mm}$ GaAs chip. The D/A converter provides the required bias voltage to the second gate of the dual-gate FET for control of the output power level. Control with a dual-gate FET has several advantages. The FET gain can be changed over a large dynamic range $(20$ to $40 \mathrm{~dB})$. Over this range the amplifier has a minimum transmission phase shift (approx. $5^{\circ}$ ), and FET input/ output impedances are essentially constant providing constant shape of the gain-frequency response curve. A mounted VPA is shown in Fig. 3. Notice the number of connections required for gain control.

\section{$30 \mathrm{GHz}$ Monolithic Receive Module}

This module combines five separate receiver functions on a single GaAs chip. The separate receiver submodule developments include a low nolse amplifier, an amplifier with gain control, a phase shifter with controls, a mixer and an IF amplifier. This $30 \mathrm{GHz}$ receive module development is in an early stage with two of the $4 \mathrm{yr}$ scheduled for this effort having been completed.

A number of advances in this receive module technology have been made under NASA contract. Hughes has fabricated a two-stage low norse amplifier with $13 \mathrm{~dB}$ gain and $6.5 \mathrm{~dB}$ noise figure. An IF amplifier with an output frequency range of 2 to $6 \mathrm{GHz}$, and an analog phase shifter and mixer have also been fabricated.

Honeywell has also made advances in the fabrication of a $30 \mathrm{GHz} 5$ bit phase shifter for the receive module; this is described elsewhere. 8 The phase shifter uses $30 \mathrm{GHz}, 0.25 \mu \mathrm{m}$ dual gate FETs. Preliminary test results show the device gain to be approximately $10 \mathrm{~dB}$. By varying the second gate bias, a range in gain adjustment of $25 \mathrm{~dB}$ has been achieved. This phase shifter requires five TTL-compatible electrical connections. A packaged phase shifter submodule with its connections is shown in Fig. 4.
From the above discussion of MMIC characteristics, it is evident that MMICs need several digital and RF connections for their operation. Optical distribution techniques which can minimize the complexity of these connections are outlined below:

\section{Optical Signal Distribution Techniques}

As a solution to the MMIC phased array signal distribution problem, the use of fiber optic technology may provide an answer. Optical fiber can be used to transmit both analog and digital signals. Other advantages include small size, lightweight, mechanical flexibulity and large bandwidth. Optical wavelength division multiplexing techniques, which allow distribution of diverse signals simultaneousiy on a single fiber, will futher reduce the signal distribution complexity. Since short links are involved in GaAs MMIC phased array signal distribution, the shorter 8500 to 9000 A wavelength will suffice. Also, GaAs-based devices required to provide the interface operate in this region. Several GaAs optical integrated circuits such as a photodiode preamplifier and a laser/field effect transistor have been demonstrated. These circuits, if integrated with GaAs MMICs, can simplify the optical interface to the MMICs.

Two possibilities for control/data signal distribution to the array modules exist. An individually modulated laser diode can be multiplexed to be the feed for all the elements. However, in large arrays this could limit how rapidly the beam could be steered due the overall length of the addressing time. Alternatively, for an array with $N$ elements, $N$ Iaser diodes could be used to distribute the signals in parallel. A combination of both distribution methods using a modulated laser drode for each column or row is also a viable option.

A trade-off between message size and bit-rate will ultimately determine which distribution scheme is optimum. Increased amplitude and phase quantization or chip complexity requires larger message lengths and the longer the message, the higher the bit-rate. Given a fixed bit-rate, parallel signal distribution could be used to produce the desired speed.

Wavelength or time multiplexing techniques can be used for control of phase and gain functions of MMICs. Rapidly altering the phase excitation of each array element to produce rapid beam steering is achieved by using an array processor to download control/data signals to the individual modules as dictated by the controller. [Synchronized data transmission of the control/data signals is used to transfer information from the array processor to each module.]

Wavelength division multiplexing (WDM) takes advantage of the characteristic of optical fiber that much of the available spectral transmission band is not used. By using sources that produce many spectral bands within the overall fiber bandwidth, simultaneous transmission of many optical signals over the same fiber can be accomplished leading to multiple transmission channels (as shown in Fig. 5). This technique, however, requires optical sources capable of producing 
multiple stable bands separated by a few tens of nanometers, crosstalk levels between -20 and $-30 \mathrm{~dB}$, and optical power sufficient to support $\mathrm{N}$ multiple channels as well as the insertion loss of the WDM components.

RF transmission requires that an identical signal be fed to all modules in parallel, which necessitates the need for an optical data bus (see Fig. 6). An optical data bus configured from transmitting star couplers seems to be a likely candidate. This type of coupler features a high efficiency, but suffers from the limitation that the number of input and output ports is fixed by initial design and is not expandable once chosen. The overall array size is then limited not only by the coupling efficiency of the star coupler, but more importantly, by the optical transmitter power output and/or receiver sensitivity. given by:

The balance equation for this type of link is $P_{S}-P_{R}=L_{S}+2 \alpha_{f} L+4 L_{C}+L_{S P}+$ SYSTEM MARGIN

(20 to 50 percent)

where,

$P_{S}$ source power, $\mathrm{dBm}$

$P_{R} \quad$ receiver sensitivity, $d B m$

$L_{S}$ insertion loss of the coupler,

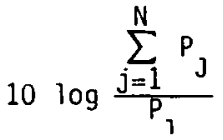

$P_{J}$ the output power from each port

$P_{i}$ the input power to the coupler

$a_{f}$ fider attention per foot

L total fiber length of link, ft

$L_{C}$ connector loss

$L_{S P}$ splitting factor, $10 \log N(N=$ number of coupler ports)

Optical Components and Link Considerations for GaAs MMICs Phased Array Signal Distribution

Signal distribution for GaAs MMIC phased arrays via optical fibers can be achieved by using integrated optoelectronic circuits on GaAs to provide an interface for the fiber, to the MMICs and the array processor. Such circuits, required for GaAs MMIC receive and transmit modules and array processor, are shown in Figs. 7 and 8.

Interfaces for phase and amplitude control of the receiver and transmitter are digital. Input RF to the transmitter, local oscillator frequency to the receiver and output from the receiver can be modulated on the optical fiber. Considerations for both digital and RF optical fiber links for MMICs are discussed below.

\section{RF Signal Distribution Considerations}

Direct, or indirect optical intensity modulation techniques can be used for distribution of the RF signal to the MMIC depending on the frequency limitation of the various optical components used in either technique. Direct intensity modulation of lasers and indirect modulation of lasers via modulators are shown in Figs. 9 and 10 , respectively.

Direct laser modulation (using GaAs/GaAlAs semiconductor laser) has been demonstrated up to $8 \mathrm{GHz}^{-10}$ and is being extended to even higher frequencies. Il However, the highest modulation frequency achrevable with this technjque is limited due to fundamental reasons. 1

The insertion loss parameters for the RF link have been obtained by Stephens, et al.12 and are given below:

$$
\text { Insertion loss }=\left[E_{m} \frac{R_{L}}{R_{S}}\left(n_{L} R_{0} \alpha_{0}\right)^{2}\right]^{-1}
$$

where $R_{L}$ and $R_{S}$ are source and load impedances, $n_{L}$ is the laser efficiency, $R_{D}$ is the detector responsivity, $\alpha_{0}$ is optical losses, and $E_{\mathrm{In}}$ is matching circuit losses. When the detected optical power is low, detector and amplifier noise usually dominates.

The conclusion regarding fiber optic signal distribution manifolds is therefore that the required $S / N$ ratios for $R F$ signal distribution in a phased array may be obtained, but that the number of output ports is severely limited, typically to about six from a single laser of the usual $10 \mathrm{~mW}$ output power.

External modulation has been demonstrated at frequenciess up to $17 \mathrm{GHz}$. By using $\mathrm{L}_{7} \mathrm{NbO}_{3}$ crystals 13 the frequency range can be further extended by various improvements. The threshold for optical power damage and the efficiency for integrated optical modulators is low. If such modulators are designed on GaAs they offer the possibility of monolithic integration.

The trade-offs to be made in considering whether a fiber optic distribution network is a viable replacement for a conventional distribution network, lies chiefly in considerations of loss and stability. In addition, fiber optic networks can carry multiple signals as well.

It has been calculated, that by using optimized values of the parameters in Eq. (1), the intrinsic insertion loss of the electrical-optical conversion could be reduced to a minimum of $10 \mathrm{~dB}$.

The overall S/N for a single optical fiber link depends on the photodetector shot and thermal noise together with thermal noise associated with the amplifier following the photodetector, and the laser intensity noise. For a signal transmission at gigahertz frequencies, laser noise is generally dominant. 
If such RF-optical fiber links are viable, lasers and photodetectors can be monolithically integrated to reduce weight, power cost, and circult parasitics. For a MMIC phased array signal distribution network, a number of 0ICs will be required. For example, for the input RF signal and local oscillator reference signals for MMIC modules using intensity modulation, the following GaAs optical integrated circuits are needed: a high frequency external modulator on GaAs with high efficiency and a high optical damage threshold; a wide band integrated photodetector and preamplifier for demodulation of the signal, and an integrated laser. In addition, a high power integrated laser capable of being directly or indirectly modulated at high frequencies with an integrated driver is needed.

To carry the IF signal from the receive module to the on-board processing system, an integrated laser and low frequency driver with extremely linear performance, and an integrated very low nolse photodetector and preamplifier on GaAs substrate are needed.

\section{Optical Interface to Digitally Controlled MMICs}

Phase and amplitude control of GaAs MMICs can be achieved via a single fiber interconnect to an array processor rather than the several electrical connections needed currently. Such an interconnect will require the monolithic integration of optical components (laser, photo diode, etc.) with GaAs MMICs and array processing chops. The recent developments in optoelectronic integrafed circuit (OEICS) technology make this feasible, 14 although their compatibility with MMIC fabrication processes have yet to be determined. NASA Lewis has taken the inftiative to integrate a photoreceiver with a MMIC. 15 If the results are successful, subsequent OEICS will be required to develop an optical interface to MMICs and an array processor as shown in Figs. 7 and 2 , respectively.

The direct optical control of phase shifting and gain functions of MMICs is also a possibility which can further simplify the MMIC/optical interface. Optical control of microwave devices and circuits has been demonstrated.16,17 However, such techniques can provide switching but not sufficient phase shifting, and monolithic integration of such methods also has to be shown.

\section{Conclusions}

Optical techniques offer several advantages for the signal distribution network for GaAs MMIC based phased array antennas. optical interfacing to digitally controlled phase and gain functions of MMICs via single fiber and optoelectronic integrated circuits is achievable. Microwave signals in some cases can be brought on via optical fibers to MMICs. However, the feasibility of these techniques depends upon the development of monolithic integration of optical components with MMIC and digital circuits. Optical distribution links with optoelectronic integrated circuits as interfaces will provide further advantages for on-board optical processing and intersatellite links for further space communication systems.

\section{References}

1. Semetana, J., "Application of MMIC Modules in Future Multiple Beam Satellite Antenna Systems," NASA TM-83344, 1982.

2. Anzic, G. and Connolly, 0.J., "20 and $30 \mathrm{GHz}$ MMIC Technology for Future Space Communication Antenna Systems," Technical 0igest 1984-GaAs IC Symposium, IEEE, New York, 1984, pp. 189-192.

3. Koepf, G.A., "Optical Processor for PhasedArray Antenna Beam Formation," Optical Technology for Microwave Applications, SPIE Vol. 477, S.-K. Yao, ed., SPIE, Bellingham, WA, 1984 , pp. 75-81.

4. Sheehan, P.G., and Forrest, J.R., "The Use of Optical Techniques for Beamforming in Phased Arrays," Optical Technology for Microwave Applications, SPIE Vol. 477, S.-K. Yao, eds., SPIE, Bellingham, WA, 1984, pp. 75-81.

5. Levine, A.M., "Fiberoptics for Radar and Data Systems," Laser and Fiber Optics Communicatrons, SPIE Vol. 150, M. Ross, ed., SPIE, Be111ngham, WA, 1978, pp. 185-192.

6. Gupta, A., Kaelin, G., Steın, R., Ip, K., Peterson, W., and Mikasa, M., "A $20 \mathrm{GHz}$ 5-Bit Phase-Shift Transmit Module with $16 \mathrm{~dB}$ Gain," Technical Digest 1984 - GaAs IC Symposium, IEEE, New York, 1984, pp. 197-200.

7. Sokolov, V., Geddes, J., Contolatis, A., Banhahn, P.E., and Chao, C., "A Ka Band GaAs Monolithic Phase Shifter, "IEEE Transactions on Microwave Theory and Technique, Vol. MTT-31, No. 12, Dec. 1983, pp. 1077-1083.

8. Saunier, P., Kim, B., and Frensley, W.K., "GaAs Integrated Digital-to-Analog Converter for Control of Power Dual-Gate FET's," Electronics Letters, Vol. 19, No. 5, Mar. 3, 1983, pp. 162-163.

9. Keiser, G., Optical Fiber Communications, McGraw-H111, New York, 1983.

10. Lau, K.Y., Bar-Chaım, N., Ury, I., Harder Ch., and Yariv, A., "Direct Amplitude Modula$t i o n$ of Short-Cavity GaAs Lasers up to X-band Frequencies," Applied Physics Letters, Vol. 43, No. 1, July 1983, pp. 1-3.

11. Lau, K.Y., Harder, Ch., and Yariv, A., "Oırect Modulation of Semiconductor Lasers at $f>10 \mathrm{GHz}$ by Low Temperature Operation," Applied Physics Letters, Vol. 44, No. 3, Feb. 1, 1984, pp. 273-275.

12. Joseph, T.R., Stephens, W., and Chen, B., "Fiber optic RF Links," Optical Technology for Microwave Applications, SPIE Vol. 477, S.-K. Yao, ed., SPIE, Bellingham, WA, 1984 , pp. $52-56$.

13. Blauvelt, $H_{.}$, and Yen, H., "Transmitter and Receiver Design for Microwave Fiber Optic Links," Optical Technology for Microwave Applications, SPIE Vol. 477, S.-K. Yao, eds. SPIE, Bellingham, WA, 1984, pp. 44-51. 
14. Carney, J.K., Helix, M., and Kolbas, R.M., "Operation of Monolithic Laser/Multiplexer Optoelectric IC," Optical Interfaces for Digital Circuits and Systems, SPIE Vol. 466 , R.A. Milano, ed., SPIE, BeTTingham, WA, 1984 , pp. 52-58.

15. Bhasin, K.B., Ponchak, G.E., and Kascak, T.J., "Monolithic Optical Integrated Control Circuitry for GaAs MMIC-Based Phased Arrays," NASA TM-87183, 1985. (to be published in Proc. SPIE Vol. 578, Paper 578-13.
16. De Salles, A.A.A., "Optical Control of GaAs MESFET' $s$ " IEEE Transactions on Microwave Theory and Technique, Vol. MTT-31, No. 10, 0ct. 1983, pp. 812-820.

17. Herczfeld, P.R., Daryoush, A.S., and Contavino, V.M., "Optically Tuned and Modulated Microwave Oscillator Circuits," First International Conference on Integrated optical Circuit Engineering, SPIE Vol. 517, 5. Sriram and O.B. Ostrowsky, eds., SPIE, Bellingham, WA, 1984, pp. 48-56. 


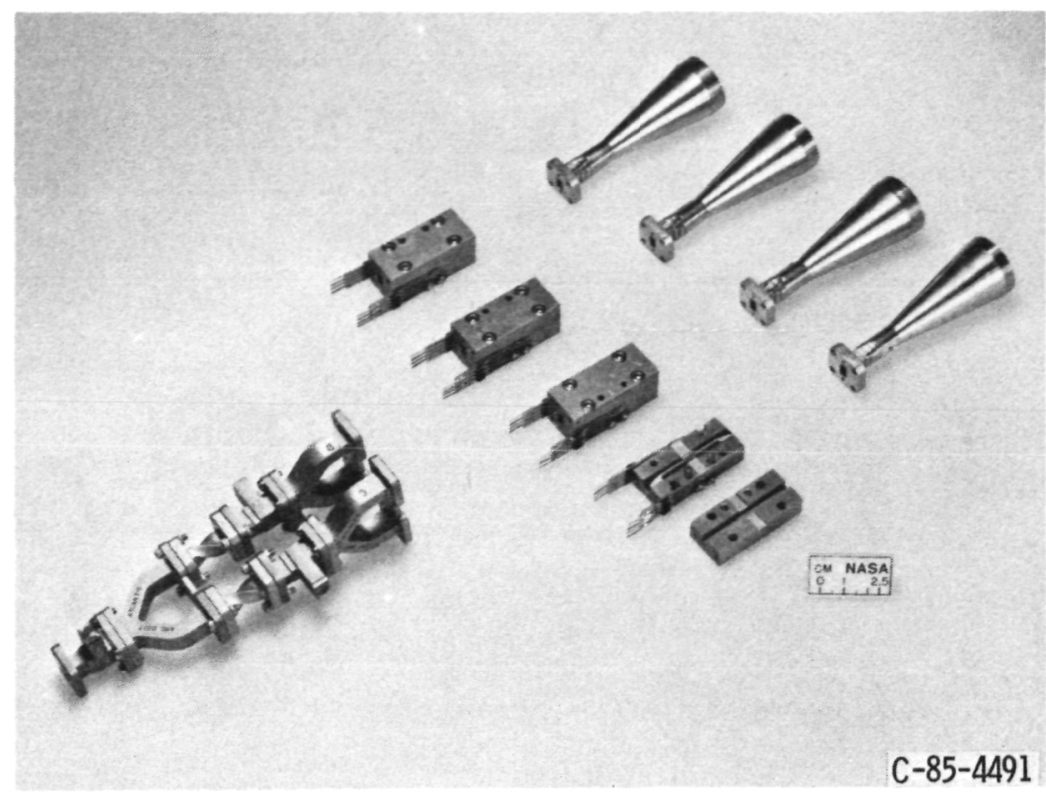

Figure 1. - Experimental four horn hybrid monolithic integrated circuit phased array feed system.

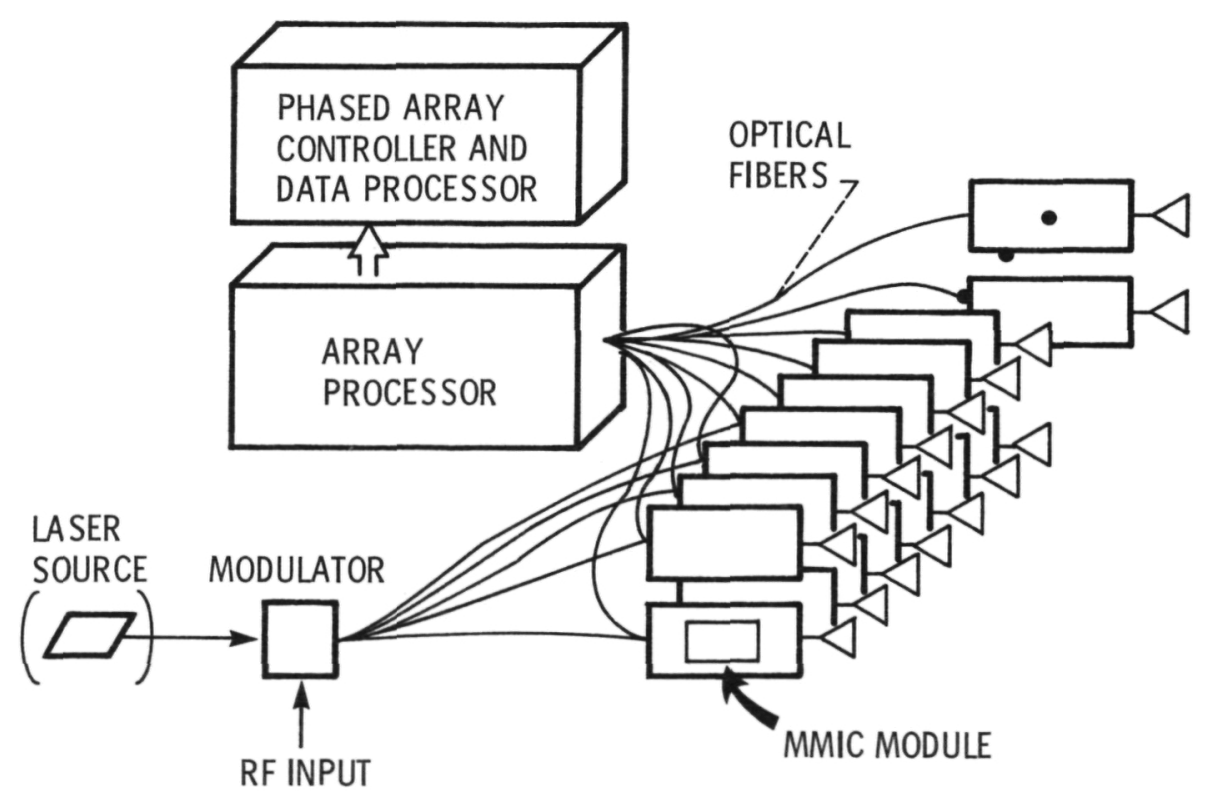

Figure 2. - Conceptual diagram of optically fed and controlled GaAs Monolithic microwave integrated circuit (MMIC) based phased array. 


\section{ORIGINAL PAGE IS \\ OF POOR QUALTTY.}

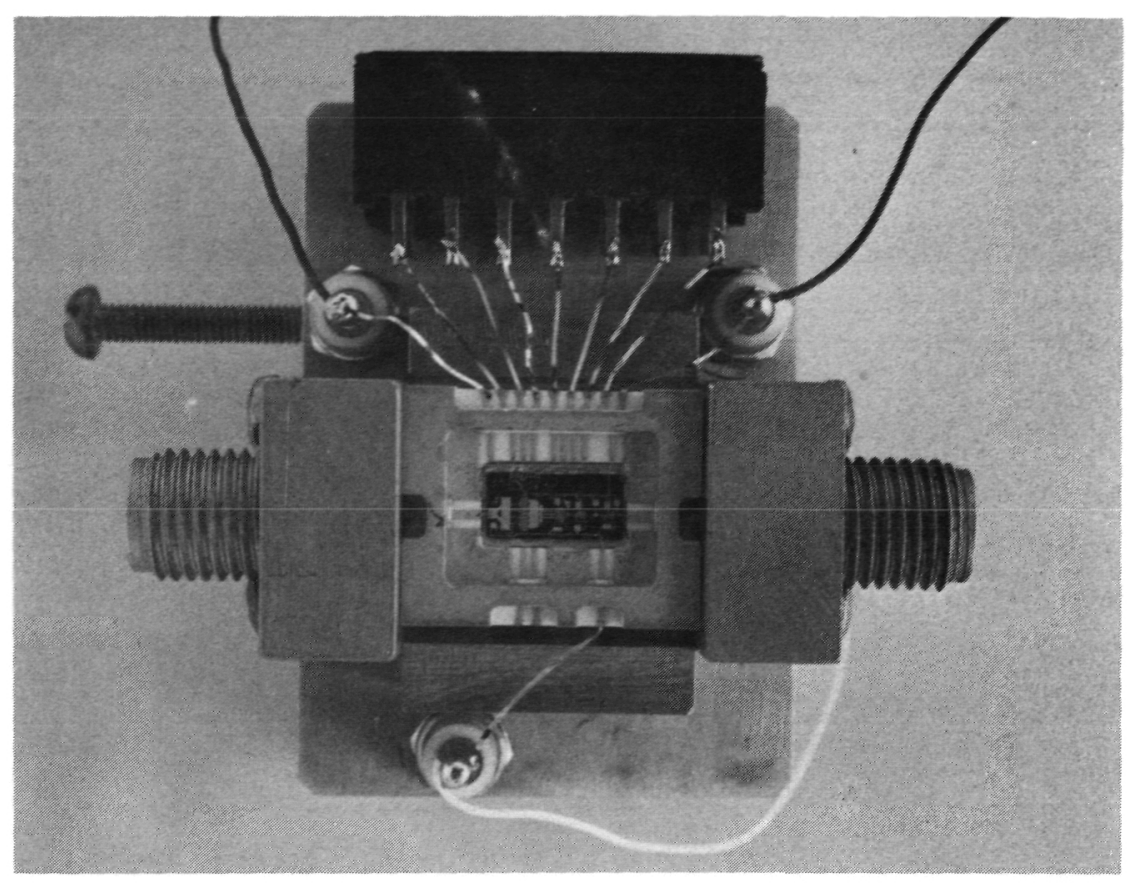

Figure 3. - MMIC variable power amplifier (VPA) module in a test fixture.

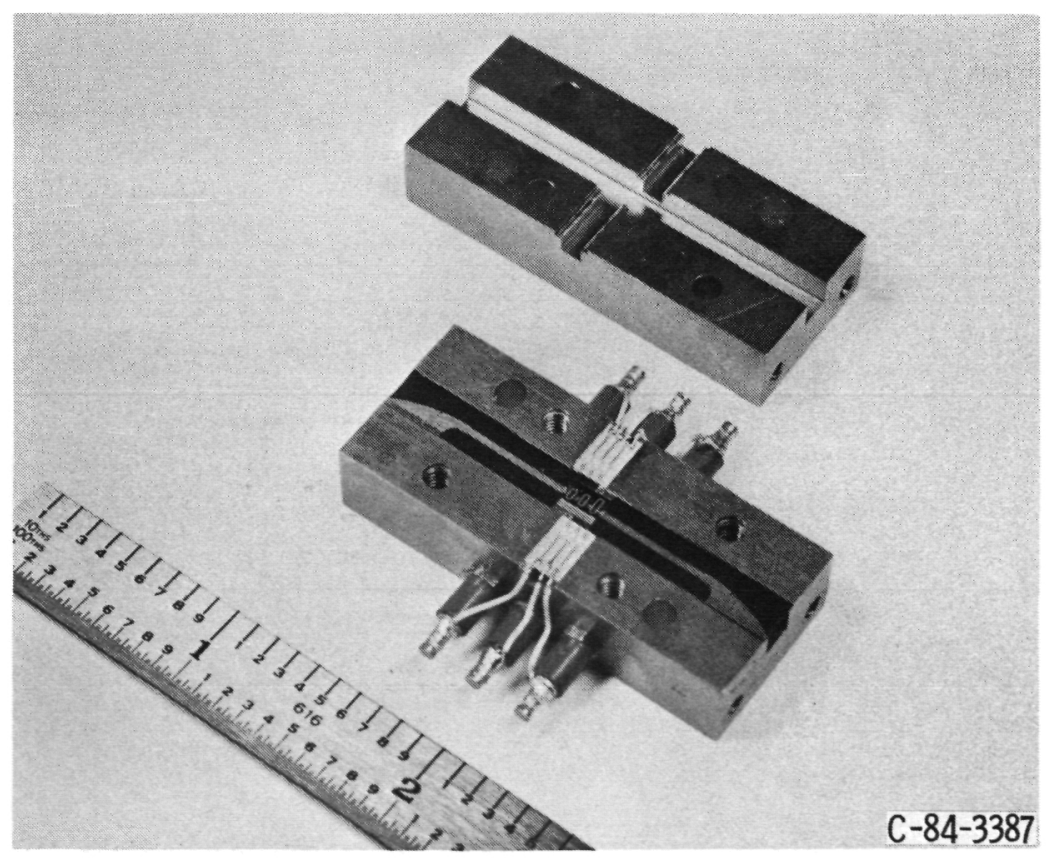

Figure 4. - MMIC variable phase shifter (VPS) module in a test fixture. 


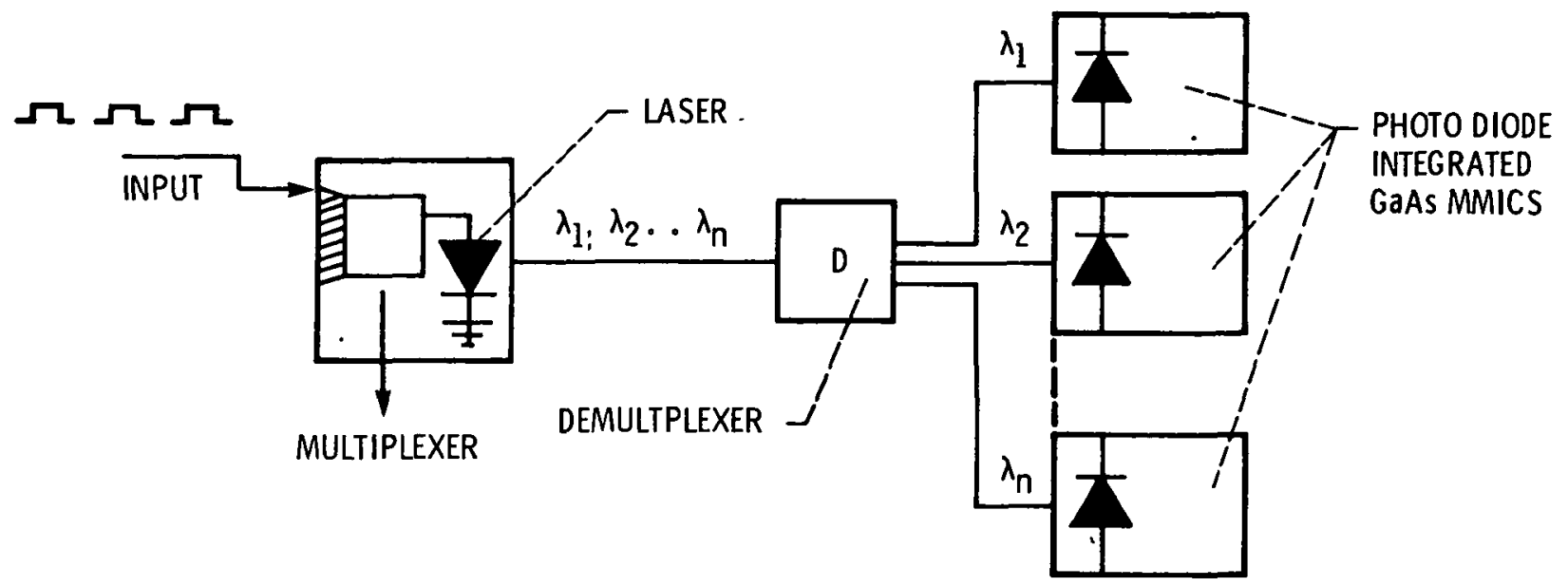

Figure 5. - Optical wavelength division multiplexing (WDM) technique for digital signal distribution.

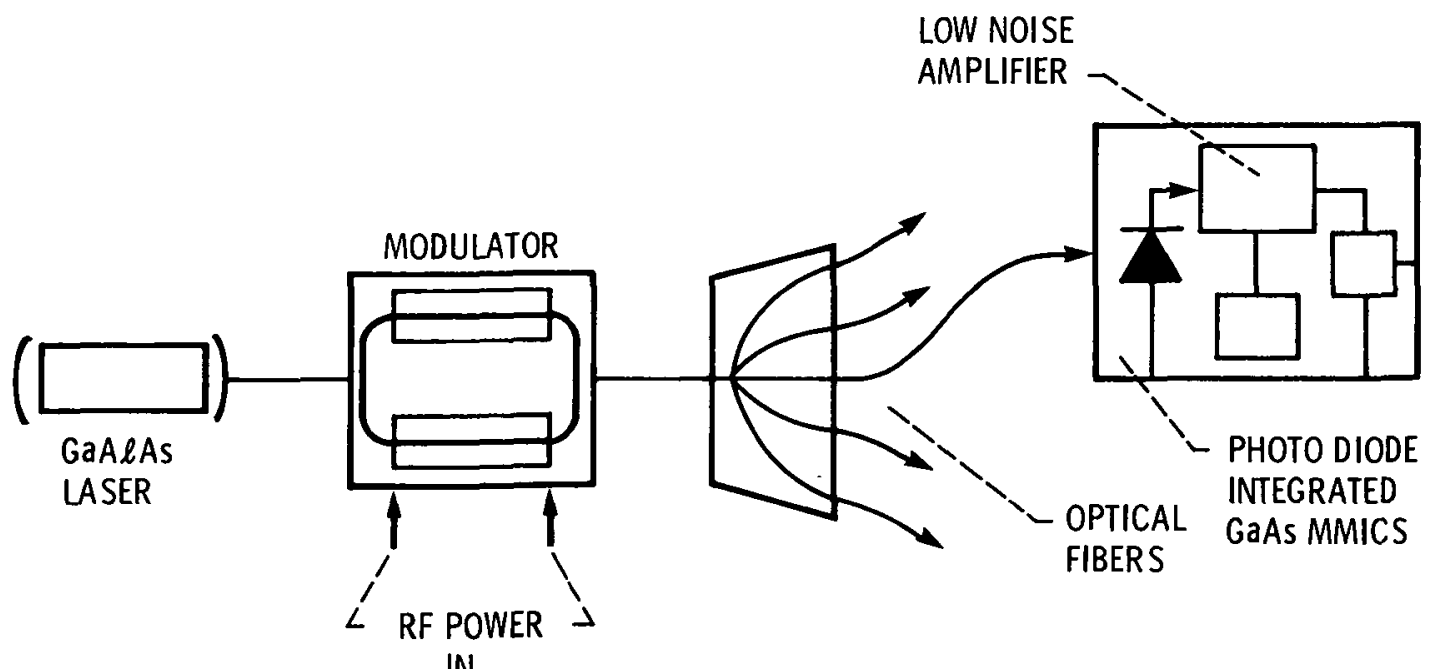

IN

Figure 6. - Optical signal distribution network for MMIC module RF signals. 


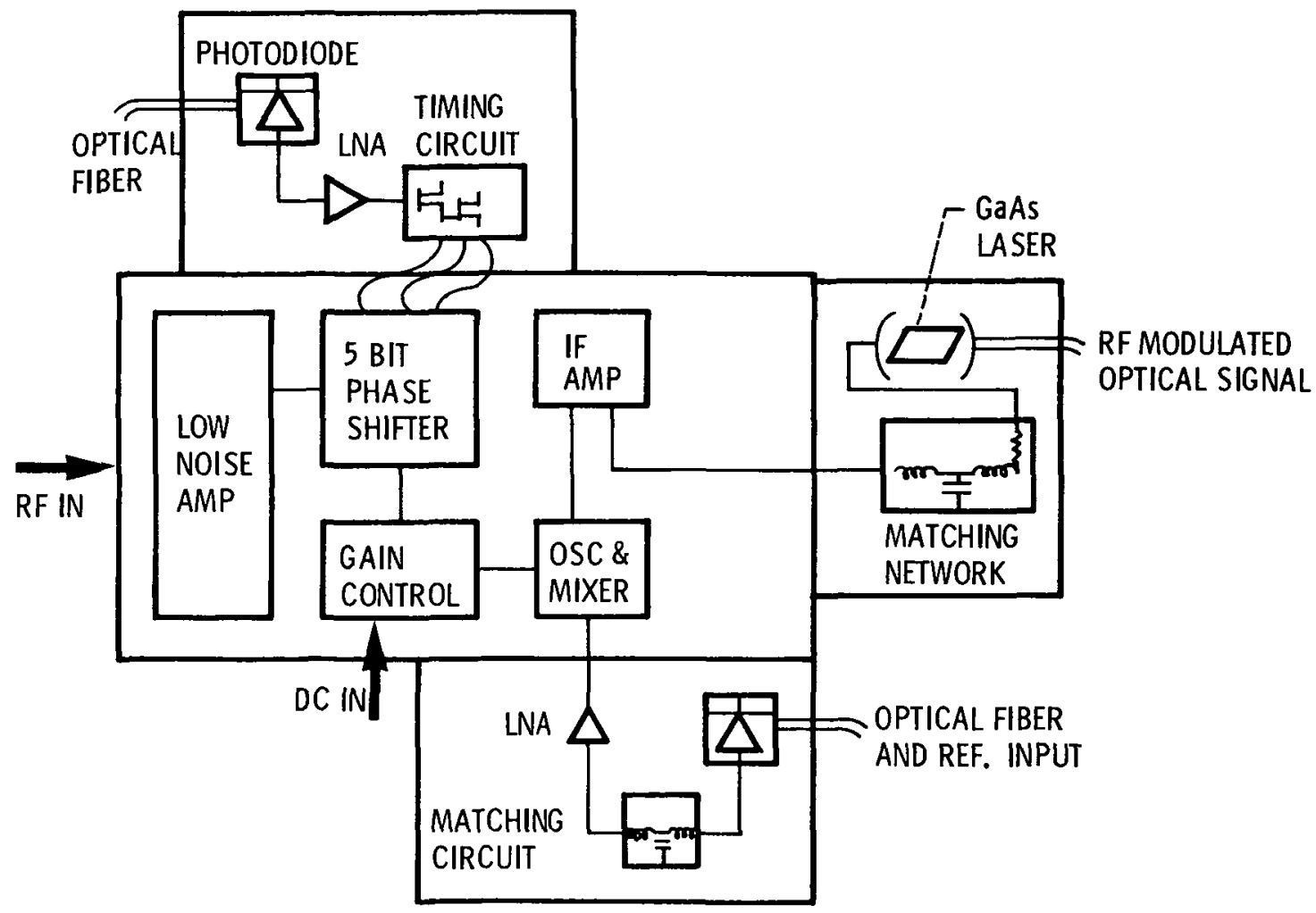

Figure 7. - Optical integrated circuits interfaced with MMIC receive module for optical fiber interconnections. 


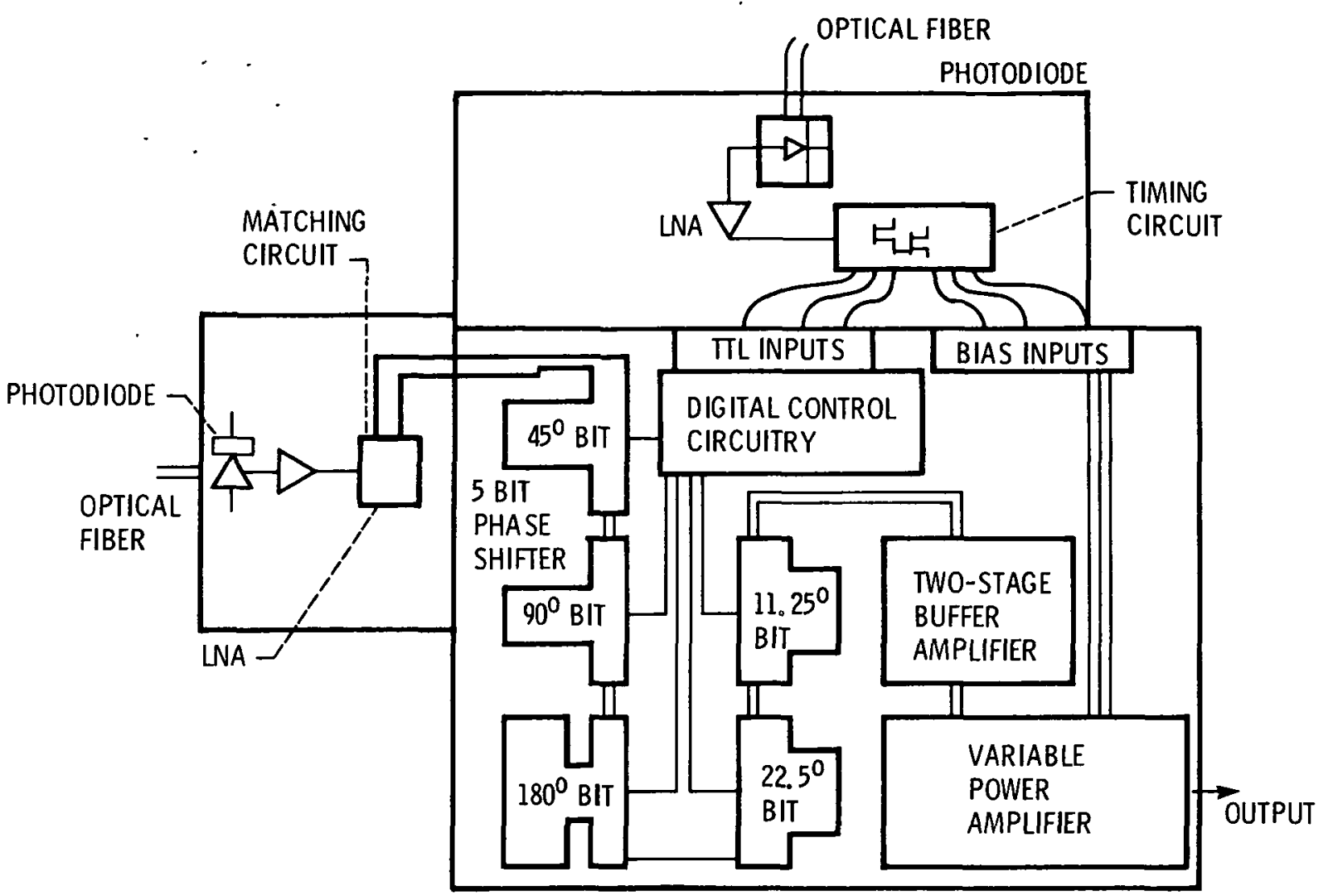

Figure 8 - Optical integrated circuits interfaced with MNIC transmit module for optical fiber interconnections. 


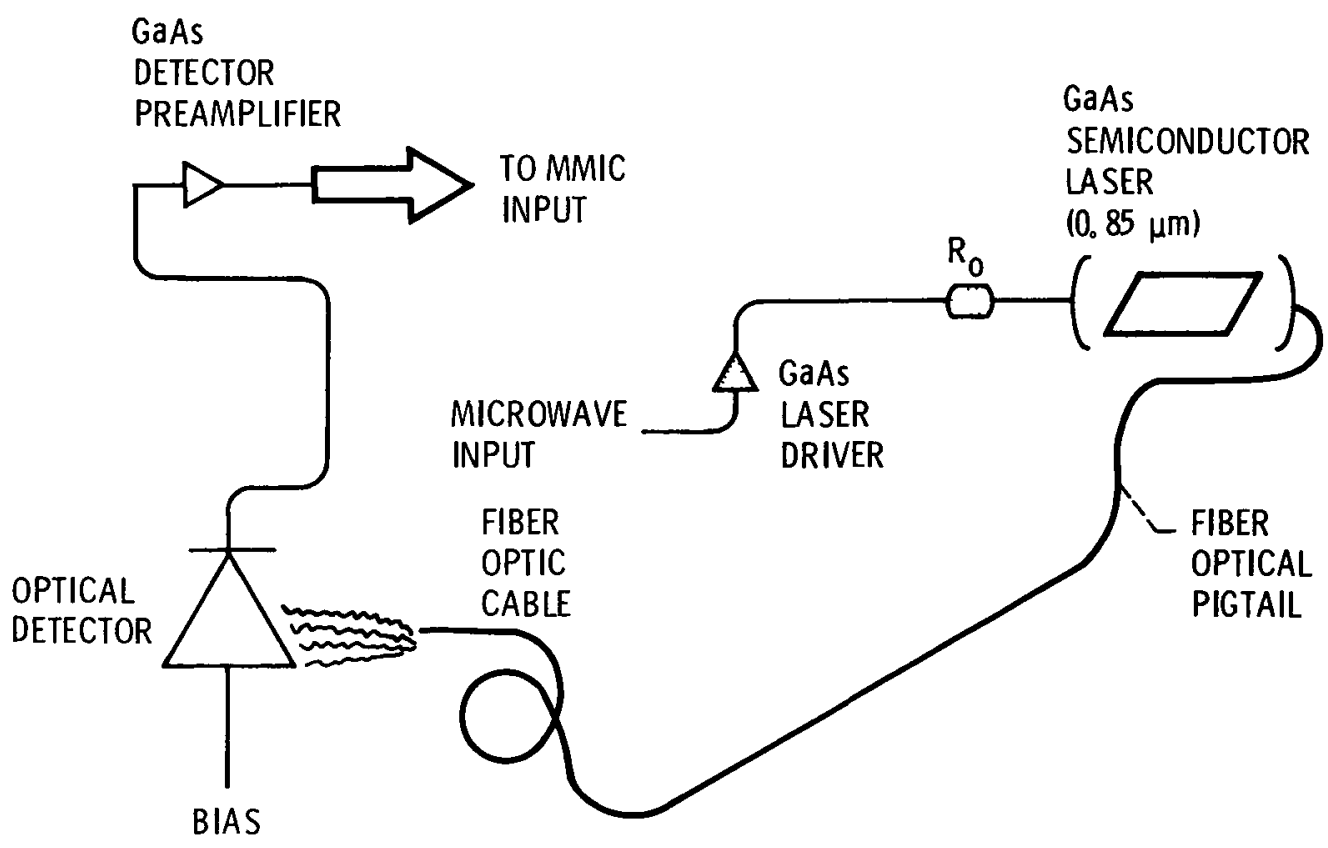

Figure 9. - Direct laser modulation techniques using GaAs components.

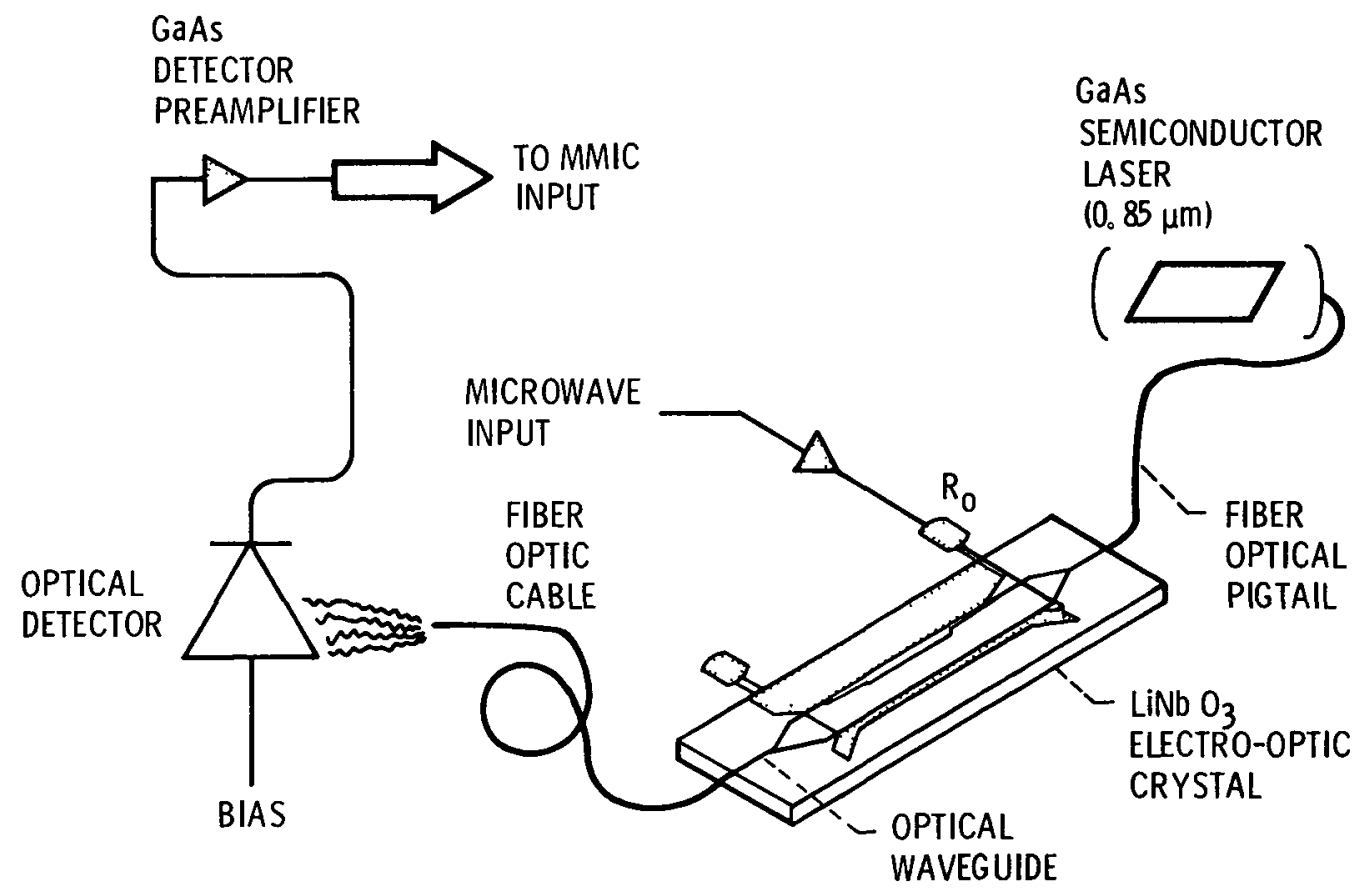

Figure 10. - Indirect laser modulation techniques using GaAs components. 


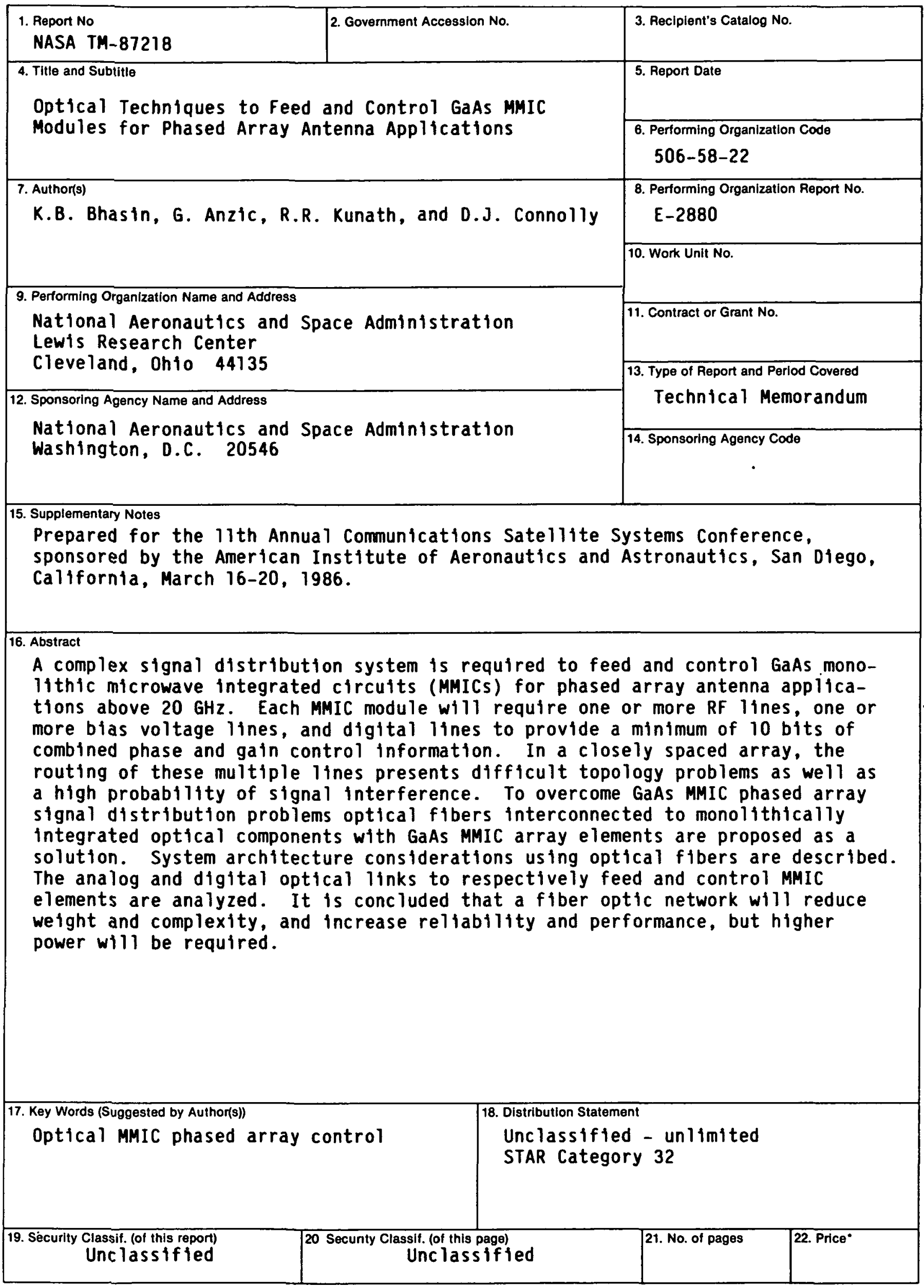

"For sale by the National Technical Information Service, Sprıngfield, Vırgınıa 22161 
Natıonal Aeronautics and Space Administration

Lewis Research Center

Cleveland. Ohio 44135

Official Business

Penalty for Private Use $\$ 300$
SECOND CLASS MAIL

ADDRESS CORRECTION REQUESTED

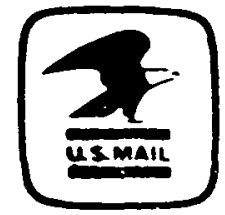

Pastage and Fees Pard National Aeronautics an:d Space Administraticn NASA-451 